\title{
Evaluation of chemiluminescence, procoagulant activity and antigen presentation by monocytes from lepromatous leprosy patients with or without reactional episodes
}

\author{
D. O. SANTOS* $ף$, P. N. SUFFYS $\dagger$, A. L. MOREIRA $\ddagger$, \\ K. BONIFACIO $\ddagger$, J. L. SALGADO $\ddagger$, D. ESQUENAZI , \\ A. L. BERTHO§ \& E. N. SARNO $\ddagger$ \\ *Department of Cellular and Molecular Biology, Federal Flumi- \\ nense University, Rio de Janeiro; $\dagger$ Biochemistry and Molecular \\ Biology Department, Oswaldo Cruz Institute, Fiocruz, Rio de \\ Janeiro; $\ddagger$ Leprosy Unit, Department of Tropical Medicine, Oswaldo \\ Cruz Foundation, Rio de Janeiro; §Protozoology Department, \\ Oswaldo Cruz Institute, Fiocruz, Rio de Janeiro, Brazil
}

\section{Accepted for publication 12 October 1993}

Summary In this study, we evaluated the activity of peripheral blood mononuclear cells (PBMC), isolated from treated and untreated lepromatous leprosy patients, from lepromatous leprosy patients during and after reactional episodes (erythema nodosum leprosum (ENL) and reversal reaction (RR), and from normal healthy individuals. We determined reactive oxygen intermediate (ROI) production, procoagulant activity (PCA) and HLA-DR antigen expression of monocytes, besides lymphoproliferation, both in the presence and absence of various stimulatory agents. Phorbol myristate acetate (PMA) stimulated ROI production by monocytes from all the groups studied, with patients during reactional episodes ( $E N L$ and $R R$ ) showing a significantly higher response $(p<0.009$ and $p<0.00001)$. Irradiated Mycohacterium leprae, although having little effect when added alone, strongly suppressed PMA-stimulated ROI production. Muramyl dipeptide (MDP) had no influence on either basal or on PMA-induced ROI production. Basal monocyte PCA, as well as $M$. leprae or concanavalin A (ConA)-induced monocyte PCA, was comparable in monocytes from all the groups studied. ConA was able to induce mitogenic activity in mononuclear cells isolated from all the groups studied. M. leprae, although stimulatory for normal individuals, did not induce lymphoproliferation in lepromatous leprosy patients, except for cells from patients during RR, which responded equally to $M$. leprae and to ConA. The absence of $M$. leprae-induced lymphoproliferation in lepromatous leprosy patients is not caused by the lack of basal HLA-DR expression, as PBMC from all individuals studied showed the same level of this antigen. Our results suggest an increase of spontaneous or PMA-

- Address for correspondence: Dr D. O. Santos, Leprosy Unit, Department of Tropical Medicine, Oswaldo Cruz Institute, Fiocruz, Rio de Janeiro, Brazil, Manguinhos, CEP 21045-900, Rio de Janeiro, RJ, Brazil. 
induced monocyte activity, as detected by ROI production, during the reactional episode; addition of $M$. leprae suppressed this response. The increase in monocyte activity could be correlated with the increase of lymphoproliferation response to $M$. leprae during RR, but not during ENL. The importance of a possible immune suppressive action of $M$. leprae is discussed.

\section{Introduction}

Leprosy, one of mankind's oldest diseases, is characterized by a very complex immunological response which determines the disease's clinical manifestations. Depending on the capacity of the infected individual to mount an adequate immune response, leprosy appears either in a paucibacillary tuberculoid form (TL), borderline forms such as borderline-tuberculoid (BT), borderline-borderline (BB), and borderline-lepromatous (BL), or in a multibacillary lepromatous stage (LL). However, leprosy is not a stable disease and is characterized by episodes of reactional states such as the reversal reaction (RR), occurring only in borderline patients, and erythema nodosum leprosum (ENL), occurring in both LL and BL patients. ${ }^{1,2}$ Cell mediated immunity (CMI) plays a key role in the immunological response to leprosy. In contrast to healthy individuals and TL patients, lepromatous leprosy patients do not mount an efficient CMI response against Mycobacterium leprae. ${ }^{3,4}$ The absence of a functional $\mathrm{T}$ lymphocyte proliferative response $^{3}$ and a low level of interferon-gamma (IFN- $\gamma$ ) production ${ }^{4}$ in response to infection with $M$. leprae probably results in adequate monocyte activation. Mononuclear phagocytes play a primary role during immune defence; functional deficiency of these cells has been observed in LL patients.

Microbicidal competence of phagocytes in leprosy patients is usually determined by measurement of reactive oxygen intermediate (ROI) production. However, probably because of the complexity of the interaction of macrophage and bacilli, conclusions from studies using this experimental design are highly contradictory. ${ }^{5-12}$ One of the initial events in the cascade of steps leading to pathogen killing in the bacterially-induced CMI response is phagocytosis of the bacilli and subsequent expression of bacterial antigens on the surface of the phagocytic cell. ${ }^{13}$ Experiments in vitro indicate that failure of antigen presentation in an immunogenic form, as observed in monocytes isolated from lepromatous leprosy patients, might lead to the lack of an efficient response. ${ }^{14}$ Monocyte activation can also be determined by measuring cellular procoagulant activity (PCA). ${ }^{15}$ $M$. leprae has been shown to induce PCA activity in monocytes from normal individuals, ${ }^{16}$ but so far no information exists about this activity in lepromatous leprosy patients.

These data prompted us to evaluate various parameters of mononuclear phagocyte activity in patients with distinct clinical manifestations of leprosy. We were able to extend our previous observations on monocyte activity from leprosy patients ${ }^{10}$ with new data on monocyte functions such as ROI production, PCA induction, HLA-DR expression, and on lymphoproliferation of mononuclear cells in vitro.

\section{Materials and methods}

SUBJECTS

All lepromatous leprosy patients were diagnosed at the Souza Araujo Ambulatory 
(Leprosy Unit, Oswaldo Cruz Foundation, RJ, Brazil). Clinical diagnosis was confirmed by skin biopsy; disease classification was according to the criteria of Ridley \& Jopling. ${ }^{17}$

Of the $59 \mathrm{LL}$ and BL patients in this study, 16 had only recently been treated, while 25 had not received any treatment. Another 14 were in a reactional episode (10 patients with ENL and 4 with RR), while 4 patients were in a postreactional state. Multidrug therapy (MDT) was as follows: monthly doses of rifampicin $(600 \mathrm{mg})$ and clofazimine $(300 \mathrm{mg})$ under supervision, as well as daily self-administered doses of dapsone (100 $\mathrm{mg})$ plus clofazimine $(100 \mathrm{mg})$ every other day for 24 months. Patients undergoing a reactional episode did not receive any specific treatment for reactions before blood collection. Patients in a postreactional state were treated by thalidomide and/or prednisone during the reactional episode. Blood collection of these patients was carried out in the first week after the reactional episode, when these patients no longer displayed any associated symptoms. The duration of the reactional episode was variable for each patient. We used 20 laboratory staff as normal controls.

\section{ISOLATION OF MONONUCLEAR CELLS}

Mononuclear leukocytes were isolated from heparinized venous blood using FicollHistopaque (Pharmacia Fine Chemicals, Piscataway, NJ, USA) as previously described. ${ }^{10}$

\section{MEASUREMENT OF ROI PRODUCTION}

We incubated 1,000,000 mononuclear leukocytes on $13 \mathrm{~mm}$ diameter glass coverslips in 24 well microtitre plates (Becton Dickinson, San Jose, CA, USA) in RPMI 1640 medium (Gibco, Grand Island, NY, USA), supplemented with 10\% foetal bovine serum (FBS)(Gibco), $2 \mathrm{mM}$ glutamine (Gibco), $100 \mathrm{U} / \mathrm{ml}$ penicillin (Gibco) and $100 \mu \mathrm{g} / \mathrm{ml}$ streptomycin (Gibco) at $37 \mathrm{C}$ in $5 \% \mathrm{CO}_{2}$. The cells were incubated with or without $20 \mu \mathrm{g} /$ $\mathrm{ml}$ irradiated, armadillo derived soluble $M$. leprae sonicate (IMM LEP BANK, Mill Hill, UK) and/or $1 \mu \mathrm{g} / \mathrm{ml}$ muramyl dipeptide (MDP; Sigma Chemical Co, St Louis, MO, USA) for $20 \mathrm{~h}$. The medium was removed and the adherent monocytes were washed 3 times with medium containing $2 \%$ FBS before use. Measurement of ROI production occurred in the presence of $50 \mu \mathrm{M}$ luminol (Sigma) as a chemiluminescence CL probe. ROI was induced by the addition of $0.5 \mu \mathrm{g} / \mathrm{ml}$ phorbol-myrystate-acetate (PMA, Sigma) and measured immediately in a Packard liquid scintillation spectrometer (model 1900 CA) as previously described. ${ }^{10}$ Results are calculated as follows: Stimulation Index (SI) $\mathrm{CL}$ after cell stimulation divided by CL of unstimulated cells. The percentages of basal CL were calculated as follows: (basal chemiluminescence $(\mathrm{CL})$ of monocytes isolated from patients minus $\mathrm{CL}$ of monocytes isolated from normal individuals divided by $\mathrm{CL}$ of monocytes isolated from normal individuals) $\times 100$.

\section{DETERMINATION OF PCA}

Mononuclear cells $\left(2 \times 10^{4} /\right.$ well $)$ were incubated in 96 well plates (Becton Dickinson) in RPMI 1640 medium, supplemented with $10 \%$ human serum in the presence or absence of $20 \mu \mathrm{g} / \mathrm{ml} M$. leprae antigen or $10 \mu \mathrm{g} / \mathrm{ml}$ ConA (Sigma). After $20 \mathrm{~h}$ the supernatant was removed and the adherent cells lysed by incubation with $100 \mu 1 n$-octyl-B-D-glucopyrano- 
side (50 $\mu \mathrm{M}$; Sigma) for $15 \mathrm{~min}$ at $37^{\circ} \mathrm{C}$ and stored at $-70^{\circ} \mathrm{C}$ until further use. PCA of the monocytes was measured using a modified Edwards \& Rickles assay. ${ }^{15}$ Briefly, cell lysates were transferred to siliconized tubes, after which $100 \mu \mathrm{l}$ of plasma was added and coagulation was induced by the addition of $\mathrm{CaCl}_{2}$ to a final concentration of $0.03 \mathrm{M}$, followed by gentle shaking. Rabbit brain thromboplastin (Baxter Healthcare Corporation, Miama, FL, USA) was used as a standard. For determination of basal monocyte PCA, aliquots of freshly isolated mononuclear cells were sedimented at $200 \mathrm{~g}$ for $5 \mathrm{~min}$ and lysed; PCA was then determined as described above.

\section{DETERMINATION OF HLA-DR ANTIGEN EXPRESSION}

At least $5 \times 10^{5}$ mononuclear cells were resuspended in $20 \mu \mathrm{l}$ of solution buffer (Earle's balanced salt solution $\mathrm{pH} 7 \cdot 2$, supplemented with $0 \cdot 1 \%$ sodium azide and $3 \% \mathrm{FBS}$ ) and incubated with OKM1 (Becton Dickinson) or anti-DR monoclonal antibodies (National Institutes of Health, Bethesda, MD, USA), followed by incubation with fluoresceinated goat antimouse Ig polyclonal antibody (Becton Dickinson). The cells were fixed with $1 \%$ paraformaldehyde and analysed in a flow cytometer FACScan (Coulter-Miami, USA).

\section{LYMPHOCYTE PROLIFERATION ASSAY}

Mononuclear cells were incubated in 96 well plates at a concentration of $2 \times 10^{5}$ cells per well in $200 \mu \mathrm{l}$ RPMI, supplemented with $10 \%$ human autologous plasma, $10 \mathrm{mM}$ HEPES, $2 \mathrm{mM}$ L-glutamine, antibiotics and $50 \mu \mathrm{M}$ 2-mercaptoethanol. Cells were stimulated with $20 \mu \mathrm{g} / \mathrm{ml} \mathrm{M}$. leprae or $15 \mu \mathrm{g} / \mathrm{ml}$ ConA (Sigma). Blast formation was determined by measuring radioactive thymidine incorporation 5 days after the addition of $M$. leprae or 2 days after the addition of ConA. Briefly, cells were labelled by the addition of $1 \mu \mathrm{Ci} /$ well of $\left[\right.$ methyl $\left.-{ }^{3} \mathrm{H}\right]$ thymidine $(5 \cdot 0 \mathrm{Ci} / \mathrm{mmol}$; Amersham, Bucks, UK) per well for $18 \mathrm{~h}$. Cells were lysed on a glass filter and the amount of incorporated labelled thymidine measured in a liquid scintillation counter. The S I was calculated as follows: incorporated radioactivity in cells stimulated with antigen divided by incorporated radioactivity in unstimulated cells.

\section{STATISTICAL ANALYSES}

Significance of the difference between the values of the various groups studied was evaluated by the Student's $t$-test. ${ }^{18}$ The $t$-test was modified to correct for the heterogeneity of variances.

\section{Results}

\section{ACTIVATION OF MONOCYTES}

Figure 1 shows the basal $\mathrm{CL}$ of monocytes isolated from normal individuals or from patients with different forms of lepromatous leprosy. Monocytes isolated from patients during ENL or RR showed a significantly higher CL versus normal individuals $(20 \%$, $p<0.0001$; and 295\%, $p<0.004$, respectively), whereas monocytes isolated from other 


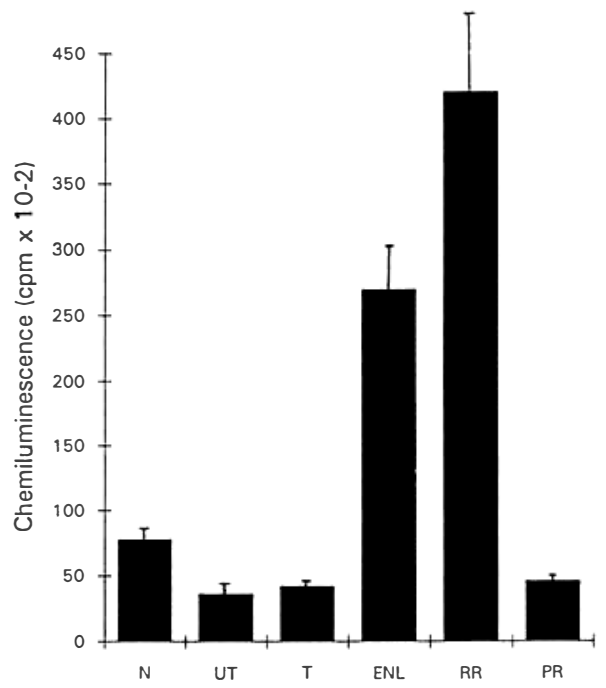

Figure 1. Basal chemiluminescence $(\mathrm{CL})$ of monocytes. Monocytes were cultured for 1 day, washed and further treated $\mathrm{f}$ or $\mathrm{CL}$ determination as described in the Materials and Methods. N, monocytes f rom normal individuals $(n=20)$; UT, untreated lepromatous leprosy patients $(n=25)$; T, treated lepromatous leprosy patients $(n=16)$; ENL, patients undergoing erythema nodosum leprosum $(n=10) ; \mathrm{R}$, patients undergoing reversal reaction $(n=4)$ and PR, patients in post-reactional state $(n=4)$.

lepromatous leprosy patients showed a basal level of CL that was lower than that found in normal individuals $(p<0 \cdot 04)$. Table 1 shows the ROI production of monocytes, isolated from normal individuals or from lepromatous leprosy patients after stimulation with PMA and $M$. leprae or MDP. Monocytes from all individuals studied showed increased ROI production after incubation with PMA. Stimulation with PMA shows a difference in ROI response for LL and BL patients. Regardless of whether BL patients were treated or not, ROI production was comparable to that of normal volunteers; LL patients showed a much lower ROI response. Patients undergoing reaction manifestations (ENL and RR) showed the strongest CL response, differing significantly from normal volunteers. Although no significant change in basal CL was found after incubation of monocytes with $M$. leprae, ROI values were repeatedly slightly lower than in the absence of $M$. leprae. Pretreatment with $M$. leprae, however, strongly inhibited the PMA-induced CL response in all groups studied. Pretreatment of cells with MDP had no influence on either the basal or PMA-induced CL response.

\section{MONOCYTE INDUCED PCA}

PCA of monocytes from normal volunteers and lepromatous leprosy patients is shown in Table 2. PCA was only detectable after co-incubation of monocytes with other mononuclear cells (data not shown). Monocytes, isolated from all groups studied, spontaneously induced PCA; values from patients in the PR state, however, were considerably lower $(p<0 \cdot 02)$. Both ConA and $M$. leprae stimulated monocyte PCA without any significant difference between the various groups studied. 
Table 1. Activation of monocytes as detected by chemiluminescence

\begin{tabular}{|c|c|c|c|c|c|c|c|}
\hline \multirow{2}{*}{$\begin{array}{l}\text { Subjects } \\
\text { Conditions at the time of the study }\end{array}$} & \multicolumn{7}{|c|}{ Chemiluminescence $^{\mathrm{a}}$} \\
\hline & PMA & $M I^{\mathrm{b}}$ & $M I+\mathrm{PMA}^{\mathrm{c}}$ & MDP & $\mathrm{MDP}+\mathrm{PMA}^{\mathrm{c}}$ & $\mathrm{MDP}+M l^{\mathrm{d}}$ & $\mathrm{MDP}+M l+\mathrm{PMA}^{\mathrm{c}}$ \\
\hline Normal individuals $(n=20)$ & $14 \cdot 0 \pm 6 \cdot 0^{c}$ & $0 \cdot 7 \pm 0 \cdot 4$ & $4 \cdot 0 \pm 3 \cdot 0$ & $0 \cdot 6 \pm 0 \cdot 3$ & $10 \cdot 0 \pm 7 \cdot 0$ & $0 \cdot 4 \pm 0 \cdot 2$ & $1 \cdot 7 \pm 0 \cdot 2$ \\
\hline $\begin{array}{l}\text { Untreated }(n=25) \\
\text { BL }^{f} \\
\text { LL }\end{array}$ & $\begin{array}{l}12 \cdot 0 \pm 4 \cdot 0\left(\mathrm{NS}^{\mathrm{g}}\right) \\
6 \cdot 0 \pm 2 \cdot 0(p<0 \cdot 01)\end{array}$ & $0 \cdot 8 \pm 0 \cdot 5(\mathrm{NS})$ & $2 \cdot 0 \pm 1 \cdot 0(\mathrm{NS})$ & $0 \cdot 7 \pm 0 \cdot 1(\mathrm{NS})$ & $10 \cdot 0 \pm 5 \cdot 0(\mathrm{NS})$ & $0 \cdot 6 \pm 0 \cdot 3(\mathrm{NS})$ & $1 \cdot 7 \pm 1 \cdot 1(\mathrm{NS})$ \\
\hline Treated $(n=16)$ & & & & & & & \\
\hline $\begin{array}{l}\text { BL } \\
\mathrm{LL}\end{array}$ & $\begin{aligned} 13 \cdot 0 \pm 3.0(\mathrm{NS}) \\
5 \cdot 0 \pm 3 \cdot 0(p<0 \cdot 01)\end{aligned}$ & $0 \cdot 5 \pm 0 \cdot 3(\mathrm{NS})$ & $1 \cdot 8 \pm 0.9(\mathrm{NS})$ & $0 \cdot 7 \pm 0 \cdot 2(\mathrm{NS})$ & $20 \cdot 0 \pm 10 \cdot 0(p<0 \cdot 02)$ & $0 \cdot 4 \pm 0 \cdot 1(\mathrm{NS})$ & $4 \cdot 2 \pm 0 \cdot 3(p<0 \cdot 02)$ \\
\hline $\operatorname{ENL}(n=10)$ & $27.0 \pm 5.0(p<0.0009)$ & $0 \cdot 6 \pm 0 \cdot 3(\mathrm{NS})$ & $3 \cdot 1 \pm 1 \cdot 5(\mathrm{NS})$ & $0 \cdot 7 \pm 0 \cdot 2(\mathrm{NS})$ & $29 \cdot 0 \pm 11 \cdot 0(p<0 \cdot 001)$ & $0 \cdot 4 \pm 0 \cdot 2(\mathrm{NS})$ & $2 \cdot 2 \pm 0 \cdot 7(\mathrm{NS})$ \\
\hline $\mathrm{RR}(n=4)$ & $32 \cdot 0 \pm 5 \cdot 0(p<0 \cdot 00001)$ & $0 \cdot 8 \pm 0 \cdot 4(\mathrm{NS})$ & $7 \cdot 2 \pm 1 \cdot 2(\mathrm{NS})$ & $0 \cdot 7 \pm 0 \cdot 2(\mathrm{NS})$ & $25 \cdot 0 \pm 8 \cdot 0(p<0 \cdot 003)$ & $0 \cdot 8 \pm 0 \cdot 3(\mathrm{NS})$ & $5 \cdot 4 \pm 0 \cdot 5(p<0 \cdot 002)$ \\
\hline $\mathrm{PR}(n=4)$ & $12 \cdot 0 \pm 6 \cdot 0(\mathrm{NS})$ & $0 \cdot 6 \pm 0 \cdot 3(\mathrm{NS})$ & $0 \cdot 9 \pm 0 \cdot 7(\mathrm{NS})$ & $1 \cdot 5 \pm 0 \cdot 7(\mathrm{NS})$ & $11 \cdot 0 \pm 7 \cdot 0(\mathrm{NS})$ & $0 \cdot 3 \pm 0 \cdot 1(\mathrm{NS})$ & $1 \cdot 1 \pm 0 \cdot 7(\mathrm{NS})$ \\
\hline
\end{tabular}

a Stimulation index $=$ chemiluminescence after stimulation/chemiluminescence without stimulation.

${ }^{\mathrm{b}} M$. leprae.

c PMA added $24 \mathrm{~h}$ addition of $M$. leprae MDP.

${ }^{\mathrm{d}} \mathrm{MDP}$ and $M$. leprae added simultaneously.

e Mean value \pm standard deviation.

${ }^{f}$ When not specified, values from BL and LL patients were not significantly different.

g Not significantly different from normal volunteers. 
Table 2. Procoagulant activity (PCA) of monocytes

\begin{tabular}{lclc}
\hline & \multicolumn{3}{c}{ PCA (mU thromboplastin) } \\
\cline { 2 - 4 } & & \multicolumn{2}{c}{ Stimulus-induced } \\
\cline { 2 - 4 } & & ConA & M. leprae \\
Subjects: conditions & Spontaneous & $1737 \pm 659$ & $1509 \pm 310$ \\
at the time of the study & $653 \pm 176^{\mathrm{a}}$ & $3038 \pm 1246$ & $2152 \pm 1208$ \\
Normal individuals $(n=20)$ & $1058 \pm 724$ & $2513 \pm 914$ & $2012 \pm 627$ \\
Untreated $(n=25)$ & $510 \pm 155$ & $2435 \pm 1088$ & $2297 \pm 1037$ \\
Treated $(n=16)$ & $1109 \pm 1154$ & $2301 \pm 568$ & $2073 \pm 291$ \\
ENL $(n=10)$ & $911 \pm 892$ & $1361 \pm 675$ & $1254 \pm 707$ \\
RR $(n=4)$ & $256 \pm 112\left(p<0 \cdot 02^{\mathrm{b}}\right)$ & & \\
PR $(n=4)$ & & & \\
\hline
\end{tabular}

${ }^{a}$ Mean value \pm standard deviation.

${ }^{b}$ All other values do not significantly differ from normal individuals.

Table 3. Flow cytometric quantification of HLA-DR expression in monocytes as measured by fluorescence

\begin{tabular}{lccc}
\hline & \multicolumn{3}{c}{ Fluorescence } \\
\cline { 2 - 4 } & $\begin{array}{c}\text { \% OKM1 } \\
\text { positive cells }\end{array}$ & $\begin{array}{c}\text { \% DR-positive } \\
\text { cells }\end{array}$ & $\%$ MFjects: conditions \\
at the time of the study & $13 \pm 7^{\mathrm{b}}$ & $16 \pm 5$ & $80 \pm 15$ \\
\hline Normal individuals $(n=20)$ & $17 \pm 8$ & $19 \pm 10$ & $77 \pm 10$ \\
Untreated $(n=20)$ & $12 \pm 1$ & $15 \pm 6$ & $75 \pm 10$ \\
Treated $(n=16)$ & $18 \pm 8$ & $16 \pm 6$ & $77 \pm 12$ \\
ENL $(n=10)$ & $17 \pm 3$ & $15 \pm 4$ & $87 \pm 14$ \\
RR $(n=4)$ & $12 \pm 4$ & $15 \pm 3$ & $81 \pm 10$ \\
PR $(n=4)$ & & & \\
\hline
\end{tabular}

a Mean fluorescence intensity of DR-positive cells.

${ }^{\mathrm{b}}$ Mean value \pm standard deviation.

HLA-DR SURFACE ANTIGEN EXPRESSION

Expression of HLA-DR by PBMC from normal individuals and lepromatous leprosy patients is shown in Table 3. No significant differences emerged between HLA-DR surface antigen expression in any of the groups studied, and the number of monocytes within the mononuclear cell population was the same, independent of the patient's clinical status.

\section{LYMPHOCYTE PROLIFERATION}

Table 4 shows that cells from all the groups studied responded to stimulation with ConA. Patients during R R showed a stronger response. Mononuclear cells, isolated from normal individuals, also proliferated strongly after incubation with $M$. leprae. Within the population of lepromatous patients, only lymphocytes isolated from patients with RR were able to respond to the addition of $M$. leprae. Post-RR patients partially retained proliferative capacity. 
Table 4. Lymphoproliferative responses of PBMCs from healthy individuals and leprosy patients

\begin{tabular}{|c|c|c|c|c|}
\hline \multirow{2}{*}{$\begin{array}{l}\text { Subjects: conditions } \\
\text { at the time of the study }\end{array}$} & \multicolumn{2}{|c|}{ ConA } & \multicolumn{2}{|c|}{ M. leprae } \\
\hline & - & + & - & + \\
\hline Normal individuals $(n=20)$ & $1024 \pm 213^{\mathrm{a}}$ & $11733 \pm 1667(11 \pm 3)^{\mathrm{b}}$ & $2057 \pm 321$ & $12342 \pm 1418(6 \pm 0 \cdot 5)$ \\
\hline Untreated $(n=20)$ & $678 \pm 136\left(\mathrm{NS}^{\mathrm{c}}\right)$ & $4493 \pm 1311(7 \pm 1)\left(\mathrm{NS}^{\mathrm{d}}\right)$ & $907 \pm 158\left(p<0 \cdot 04^{c}\right)$ & $1394 \pm 300(2 \pm 1)\left(p<0.02^{\mathrm{d}}\right)$ \\
\hline Treated $(n=12)$ & $708 \pm 88$ (NS) & $2665 \pm 199(4 \pm 1)(p<0 \cdot 001)$ & $1325 \pm 330(p<0.04)$ & $1380 \pm 302(1 \pm 2)(p<0.001)$ \\
\hline $\operatorname{ENL}(n=10)$ & $341 \pm 67(p<0.04)$ & $3955 \pm 924(11 \pm 0 \cdot 5)(\mathrm{NS})$ & $842 \pm 218(p<0.04)$ & $757 \pm 125(1 \pm 1)(p<0.001)$ \\
\hline $\mathrm{RR}(n=4)$ & $478 \pm 41(p<0.04)$ & $6597 \pm 68(13 \pm 1)(\mathrm{NS})$ & $369 \pm 43(p<0.0001)$ & $1845 \pm 328(5 \pm 1)(\mathrm{NS})$ \\
\hline \multicolumn{5}{|l|}{$\operatorname{PR}(n=4)$} \\
\hline ENL & $405 \pm 51(p<0 \cdot 001)$ & $2638 \pm 804(7 \pm 0 \cdot 8)(\mathrm{NS})$ & $412 \pm 53(p<0.001)$ & $824 \pm 126(2 \pm 1)(p<0.001)$ \\
\hline $\mathrm{RR}$ & $378 \pm 137(p<0.001)$ & $3529 \pm 1204(9 \pm 1)(\mathrm{NS})$ & $525 \pm 75(p<0.001)$ & $1785 \pm 148(3 \pm 2)(p<0.001)$ \\
\hline
\end{tabular}

${ }^{a}$ Mean value in $\mathrm{cpm} \pm$ standard deviation.

${ }^{\mathrm{b}}$ Stimulation index $=$ mean $\mathrm{cpm}$ upon stimulation/mean $\mathrm{cpm}$ without stimulation

${ }^{c} p$ value as compared to normal individuals.

${ }^{\mathrm{d}} p$ value as compared to the stimulation index of normal individuals. 


\section{Discussion}

Previous studies on ROI production by phagocytes from healthy individuals and lepromatous leprosy patients, upon stimulation with $M$. leprae and other agents, are contradictory. ${ }^{5-9,11,12} \mathrm{We}$ therefore measured various parameters of monocyte activity in the presence or absence of $M$. leprae from patients at given stages along the clinical spectrum of leprosy and its reactional states.

In monocytes isolated from all individuals studied, PMA induced a marked production of ROI, a process that was much more pronounced during reactional episodes. Lepromatous patients without reactional episodes displayed a weak PMAinduced $C L$ response in comparison with normal individuals, as observed previously. ${ }^{10}$ The PMA-induced ROI production observed in patients with ENL and RR could be linked to excessive monocyte activity. The high basal ROI seen in patients during reactional episodes strengthens this hypothesis, and the presence of extravascular complex in ENL could play an important role in monocyte activation. ${ }^{1}$ No difference in ROI production was found in patients under treatment or not, an observation that could be generalized for all monocyte activities measured in this study. Furthermore, the CL response of LL patients was significantly lower than that of BL patients, who produced ROI to the same extent as did normal volunteers. This suggests some residual monocyte activity in BL patients - a finding that has recently been suggested by us when comparing monocyte activity from TL and LL patients. ${ }^{10}$

Although some groups have proposed a slow and weak ROI stimulatory capacity of $M$. leprae, ${ }^{7.11,12}$ several reports claim $M$. leprae to be devoid of any stimulatory activity. ${ }^{6,19}$ Such variable results could be partly explained by suppression of monocyte function by some fractions of $M$. leprae. We therefore tested whether $M$. leprae was capable of inhibiting the PMA-induced ROI response and, if so, to what degree this would vary in patients with different clinical forms of leprosy. Indeed, M. leprae was found to be strongly inhibitory, to a comparable extent throughout the whole gamut of leprosy patients studied. A suppressor role of various mycobacteria in skin-test responses, and an active role of suppressor factors in the so-called immune defect in LL patients, has been considered. ${ }^{20}$ Phenolic glycolipid 1 (PGL1) is unique to $M$. leprae and was recently shown to decrease superoxide anion production by monocytes from normal donors stimulated with $M$. leprae. ${ }^{11}$ It also acts as a scavenger of reactive oxygen species, ${ }^{21}$ which may contribute to protecting the bacilli from killing by its host. PGLl treatment does not inhibit PMA induced ROI production, ${ }^{11}$ so it is unlikely that PGL1 alone is responsible for the suppression observed in this study. Synergistic suppression of PGL1 and other components such as lipoarabinomannan (LAM) is not unlikely. LAM has been attributed to have both macrophage stimulatory ${ }^{22}$ and inhibitory ${ }^{23}$ capacity. In addition, delipified $M$. leprae antigen seems to restore the ability of macrophages from leprosy patients to kill the bacteria. ${ }^{24} \mathrm{M}$. leprae could also interfere with monocyte activity by inducing the release of prostaglandin E2, a well-known monocyte inhibitor. ${ }^{25}$

MDP is a synthetic analogue of Gram-positive bacterial cell wall peptidoglycan. According to Pabst \& Johnston, ${ }^{26}$ MDP retains adjuvant properties and primes macrophages to respond with increased ROI production af ter stimulation with PMA. In agreement with these findings, we observed that MDP exposure did not, in itself, induce ROI production by normal monocytes. However, MDP was unable to influence PMAinduced CL. These contradictory findings could be explained by the fact that the total 
mononuclear cell population was incubated with MDP in our experiments. As MDP itself is a synthetic analogue of peptidoglycan, and $M$. leprae suppressed the PMA-induced ROI response when incubated with PBMC under the same conditions as MDP, we can exclude the possibility that peptidoglycan fragments present in $M$. leprae suppress the $C L$ response.

PCA induced by monocyte thromboplastin was also used as a marker for monocyte activity. Monocytes induce an increase in PCA when activated by endotoxin, mitogens, antigens, or cytokines in the absence of lymphocytes. ${ }^{27}$ In our study, $M$. le prae was able to induce monocyte PCA in normal individuals, a finding previously reported by Lyberg et $a l .{ }^{16} \mathrm{MDP}$ also induced monocyte PCA in all individuals studied (data not shown). Surprisingly, PCA activity of monocytes, isolated from a PR patient, was significantly lower than in the other individuals studied. Perhaps the specific treatment for reaction episodes is suppressing this monocyte activity. Although PCA response to $M$. leprae and PMA was significant and comparable after $20 \mathrm{~h}$ of culture, we cannot exclude that PCA is more pronounced or variable between the groups studied at other time points. However, there are conflicting reports as to the time of maximal PCA after stimulation of monocytes; data ranging from 4 to $24 \mathrm{~h}$ have been reported. ${ }^{16.28}$ As co-incubation of monocytes seems to sustain the PCA response, and our measurements were performed under these conditions, PCA was determined after $20 \mathrm{~h}$.

There are many reports that suggest that patients with lepromatous leprosy are unable to exert a complete CMI response due to failure of their macrophages to present $M$. leprae antigens in an immunogenic form. ${ }^{14}$ We could not find any difference in basal HLA-DR antigen expression between PBMC from normal individuals and any of the lepromatous leprosy patients. Our preliminary results indicate that stimulation with $M$. leprae has no influence on HLA-DR expression (data not shown). Comparable levels of HLA-DR expression between normal individuals, tuberculoid and long-term treated lepromatous leprosy patients were reported in another study. ${ }^{29}$ If basal HLA-DR expression is a prerequisite for antigen presenting cells (APC) to present immunogenic $M$. leprae antigens, then, at least within this part of the complex CMI response network, APC from lepromatous patients seem to be equally functional. Native antigen presentation does not seem to be sufficient to allow induction of T-cell proliferation in response to M. leprae, as we found no increase in T-cell response after incubation of PBMC from lepromatous leprosy patients without reactional manifestations with $M$. leprae. It has been reported that the levels of HLA-DR expression of lepromatous patients reaches that of normal volunteers after stimulation with a combination of $M$. leprae and IFN- $\gamma,{ }^{29}$ without inducing lymphoproliferation.

A $\mathrm{T}$-cell proliferation response towards ConA was seen in all experimental groups studied. It has been demonstrated that the absence of T-cell responses in lepromatous leprosy patients is antigen specific. ${ }^{3}$ Indeed, when observed, lymphoproliferation of cells from lepromatous leprosy patients after stimulation with $M$. leprae was smaller than with Con A. M. leprae was, however, as potent as Con A in stimulating cells from normal individuals. This is in contrast to the results of Molloy et al. ${ }^{30}$ who did not observe a mitogenic response of mononuclear cells from normal individuals to $M$. leprae, which they ascribed to a generalized suppressive effect of contaminating LPS in their antigen preparation. In our study, neither treated nor untreated lepromatous leprosy patients showed a lymphoproliferative response towards $M$. leprae. The effect of treatment of lepromatous leprosy patients on CMI is still controversial; data obtained in our 
laboratory demonstrate an increase in LTT after long-term treatment. ${ }^{31}$ In contrast to this earlier study, the majority of our patients studied here were under drug treatment for only a short time. During RR, significant lymphoproliferation in response to $M$. leprae was observed. We also confirmed our earlier observations ${ }^{10}$ that this is not the case in patients during ENL. This could be coupled with the observation of an improvement in clinical status of the patient during and after RR, ${ }^{32}$ which probably does not occur during ENL. LTT responses were still elevated in the post-RR.

Recently, it has been proposed that the inhibitory effects of $M$. leprae for various cell functions can be correlated with the content of LPS in the preparations of M. leprae and its derivatives. ${ }^{30}$ Although the LPS content in our $M$. leprae preparation might be sufficient for some of the effects observed in this study, lipids from $M$. leprae may have LPS-like activity. Sibley et al. $^{23}$ have demonstrated the ability of LAM to inhibit macrophage activation with a potency comparable to that of LPS. Furthermore, LPS has been shown to stimulate ROI production, ${ }^{26}$ an activity that was not shared with our $M$. leprae preparation. Also, M. leprae induced a mitogenic response in mononuclear cells isolated from normal individuals and some leprosy patients. If LPS contamination in $M$. leprae preparations is involved in the suppression of mitogenic responses, it would have to be selective for cells from lepromatous leprosy patients.

\section{Acknowledgments}

This investigation received financial support from UNDP/World Bank/WHO Special Programme for Research and Training in Tropical Disease (TDR) and the CNPq. We thank Dr C. A. Mandarim-de-Lacerda for statistical analysis and the staff of the Souza Araujo Ambulatory at the Oswaldo Cruz Institute, Fiocruz for patient care and providing us with the clinical data. We also thank F. and C. Shapiro for critically reading the manuscript.

\section{References}

1 Ridley MJ, Ridley DS. The immunopathology of erythema nodosum leprosum: the role of extravascular complexes. Lepr Rev, 1983; 54: 95-107.

2 Sehgal VN. Reaction in leprosy. Clinical aspects. Int J Dermatol, 1987; 26: 278-85.

${ }^{3}$ Godal T, Mykestad B, Samuel DR, Myrvang B. Characterization of the cellular immune defect in lepromatous leprosy: a specific lack of circulating M ycobacterium leprae reactive lymphocytes. Clin exp Imm, 1971; 9: 821-31.

4 Nogueira N, Kaplan G, Levy E, Sarno EN, Kushner P, Granelli-Piperno A, Vieira L, Gould CV, Levis W, Steinman R, Yip YK, Cohn ZA. Defective interferon production in leprosy. Reversal with antigen and interleukin 2. J Exp Med, 1983; 158: 2165-170.

5 Sharp AK, Banerjee DR. Hydrogen peroxide and superoxide production by peripheral blood monocytes in leprosy. Clin Immunol, 1985; 60: 203-6.

${ }^{6}$ Holzer TJ, Kenrad EN, Victoria S, Crisper RG. Mycobacterium leprae fails to stimulate phagocytic cell superoxide anion generation. Infect Immun, 1986; 51: 514-20.

7 Holzer TJ, Hizlaitis L, Vachula M, Wafaver CW, Andersen BR. Human phagocytic cell responses to Mycobacterium leprae and Mycobaterium bovis bacillus Galmette-Guerin. An in vitro comparison of leprosy vaccine components. J Immunol, 1989; 141: 1701-1708.

8 Horwitz MA, Levis WR, Cohn ZA. Defective production of monocyte-activating cytokines in lepromatous leprosy. J Exp Med, 1984; 159: 666-78.

9 Marolia J, Mahadevan PR. Reactive oxygen intermediates inactivate Mycobacterium leprae in the phagocytes from human peripheral blood. Int J Lepr, 1989; 57: 483-91. 
10 Santos DO, Salgado JL, Moreira AL, Sarno EN. Reactive oxygen intermediates in the phagocytes from leprosy patients: correlation with reactional states and variation during treatment. Int J Le pr, 1992; 60: 92-4.

1 Vachula M, Holzer RJ, Kizaitis L, Andersen BR. Effects of Mycobacterium leprae phenolic glycolipid-1 on interferon-gamma of monocyte oxidative responses. Int J Le pr, 1990; 58: 342-46.

12 Vachula M, Worobec S, Andersen BR. A comparison of monocyte oxidative responses in leprosy patients and healthy subjects as influenced by mycobacterial lipid pretreatment. Int J Lepr, 1990; 58: 534-39.

13 Unanue ER, Cerottini JC. Antigen presentation. Faseb J, 1989; 3: 2496-502.

14 Hirschberg $\mathrm{H}$. The role of macrophages in the lymphoproliferative response to $M$. leprae in vitro. Clin exp Imm, 1978; 34: 46-51.

15 Edwards RL, Rickles FR. The role of human T cells (and T cell products) for monocyte tissue factor generation. J Immunol, 1980; 125: 606-11

16 Lyberg T, Closs O, Prydz H. Effect of purified protein derivative and sonicates of Mycobacterium leprae and Mycobacterium bovis $\mathrm{BCG}$ on thromboplastin response in human monocytes in vitro. Infect Immun, 1982; 38: 855-59.

17 Ridley DS, Jopling WH. Classification of leprosy according to immunity. A five group system. Int J Lepr, 1966; 34: 255-63.

18 Ipsen J, Feigl P. The standard deviation and other measures of variation. 1970. In: Bancroft's Introduction to Biostatistics. 2nd ed., pp. 42-64.

19 Launois P, Maillere B, Dieye A, Sarthou JL, Bach MA. Human phagocyte oxidative burst activation by BCG, $M$. leprae, and atypical mycobacteria: Def ective activation by $M$. leprae is not reversed by interferon- $\gamma$. Cell Immunol, 1989; 124: 168-74.

20 Nye PM, Stanford JL, Rook GAW, Lawton P, MacGregor M, Reily C, Humber D, Orege P, Revanker CR, Terencio de las Aguas J, Torres P. Suppressor determinants of mycobacteria and their potential relevance to leprosy. Lepr Rev, 1986; 57: 147-57.

21 Chan J, Fujiwarw T, Brennan P, McNeil M, Turco SJ, Sibille JC, Snapper M, Aisen P, Bloom B. Microbial glycolipids: possible virulence factors that scavenge oxygen radicals. Proc Natl Acad Sci USA, 1989; 86: 2453-457.

22 Barnes PF, Chatterjee D, Brennan PJ, Rea TH, Modlin RL. Tumor necrosis factor production in patients with leprosy. Infect Immun, 1988; 60: 1441-1446.

${ }^{23}$ Sibley LD, Hunter SW, Brennan PJ, Krahenbuhl JL. Mycobacterial lipoarabinomannan inhibits gamma interferon-mediated activation of macrophages. Infect Immun, 1988; 56: 1232-1236.

24 Marolia J, Robinson P, Mahadevan PR. A complex component modulating immune-deficient cells in leprosy patients leading to loss of viability of Mycobacterium leprae-a possible vaccine. Clin exp Imm, 1990; 79: 7-14.

25 Ridel PR, Jamet T, Robin Y, Bach MA. Interleukin 1 released by blood-monocyte derived macrophages from patients with leprosy. Infect Immun, 1986; 52: 303-8.

${ }^{26}$ Pabst MJ, Johnston Jr RB. Increased production of superoxide anion by macrophages exposed in vitro to muramyl dipeptide or lipopolysaccharide. J Exp Med, 1980; 15: 101-14.

27 Szabo G, Miller-Graziano C. Induction and regulation of monocyte procoagulant activity. Transplantation, 1990; 50: 301-9.

28 Rickles FR, Hardin JA, Pitlick FA, Hoyer LW, Conrad ME. Tissue factor activity in lymphocyte cultures from normal individuals and patients with hemophilia. J Clin Invest, 1973; 52: 1427-43.

29 Desai S, Birdi TJ, Antia NH. Correlation between macrophage activation and bactericidal function and Mycobacterium leprae antigen presentation in macrophages of leprosy patients and normal individuals. Infect Immun, 1989; 57: 1311-1317.

30 Molloy A, Gaudernack G, Levis WR, Cohn ZA, Kaplan G. Suspension of T-cell proliferation by Mycobacterium leprae and its products: the role of lipopolysaccharide. Proc Natl Acad Sci USA, 1990; 87: 973-77.

31 Esquenazi D, Sampaio EP, Moreira AL, Gallo ME, Almeida JA. Effect of treatment on immune responsiveness in lepromatous leprosy patients. Lepr Rev, 1990; 61: 251-57.

32 Laal S, Mishra RS, Nath I. Type I reactions in leprosy-heterogenity in T-cell functions related to the background leprosy type. Int J Lepr, 1987; 55: 481-84. 